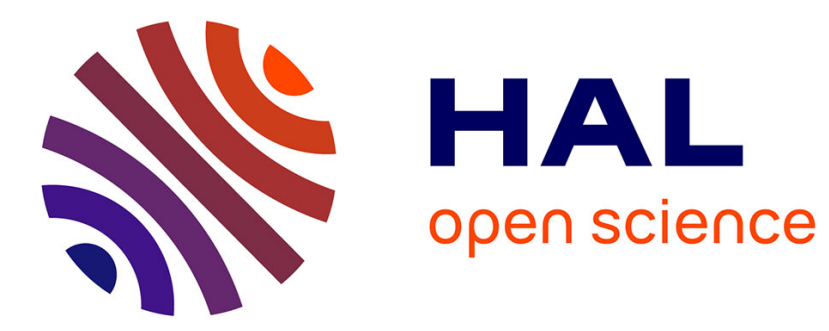

\title{
Magnetic random access memories (MRAM) beyond information storage
}

\author{
Ricardo C. Sousa, A. Chavent, V. Iurchuk, L. Vila, U. Ebels, B. Dleny, G. Di \\ Pendina, G. Prenat, J. Langer, J. Wrona, et al.
}

\section{- To cite this version:}

Ricardo C. Sousa, A. Chavent, V. Iurchuk, L. Vila, U. Ebels, et al.. Magnetic random access memories (MRAM) beyond information storage. 2020 IEEE Symposium on VLSI Technology, Jun 2020, Honolulu, United States. 10.1109/VLSITechnology18217.2020.9265053 . hal-03111373

\section{HAL Id: hal-03111373 \\ https://hal.science/hal-03111373}

Submitted on 23 Apr 2021

HAL is a multi-disciplinary open access archive for the deposit and dissemination of scientific research documents, whether they are published or not. The documents may come from teaching and research institutions in France or abroad, or from public or private research centers.
L'archive ouverte pluridisciplinaire HAL, est destinée au dépôt et à la diffusion de documents scientifiques de niveau recherche, publiés ou non, émanant des établissements d'enseignement et de recherche français ou étrangers, des laboratoires publics ou privés. 


\title{
Magnetic random access memories (MRAM) beyond information storage
}

\author{
R.C. Sousa ${ }^{1}$, A. Chavent ${ }^{1}$, V. Iurchuk ${ }^{1}$, L. Vila ${ }^{1}$, U. Ebels ${ }^{1}$, B. Dieny ${ }^{1}$, G. di Pendina ${ }^{1}$, G. Prenat ${ }^{1}$, J. \\ Langer $^{2}$, J. Wrona ${ }^{2}$, I.L. Prejbeanu ${ }^{1}$ \\ ${ }^{1}$ Univ. Grenoble Alpes / CEA / CNRS, Spintec, Grenoble, France, ${ }^{2}$ Singulus Technology, Kahl am Main, Germany \\ e-mail: ricardo.sousa@cea.fr / Phone: (+33) 438784895
}

\begin{abstract}
Magnetic random access memory (MRAM) is now available as embedded memory from major CMOS foundries. In this study, we demonstrated that slightly modified magnetic tunnel junctions than those used in conventional STT-MRAM can be used for multifunctional purposes, namely magnetic field sensing and RF oscillators. For that, the $\mathrm{FeCoB}$ storage layer thickness in the perpendicular anisotropy magnetic stack was adjusted to 1.3-1.4 nm, closer to the transition region from perpendicular to in-plane anisotropy. Two possible configurations of magnetic field sensing using the same stack can be used, achieving high sensitivity in small field range or lower sensitivity in large field range. Additionally, RF oscillator $\mathrm{GHz}$ detection and generation were also demonstrated. Further applications of this multifunctional stack can be envisioned including non-volatile and reprogrammable logic, special functions such as random number generator and memristors.
\end{abstract}

Keywords: MTJs, MRAM, embedded magnetic sensors, RF oscillators, neuromorphic

\section{Introduction}

Scaling of microelectronic circuits is facing physical limits. Extreme miniaturization of devices leads to large leakage currents responsible for inefficient power consumption, heat dissipation and reliability issues. Logic circuits making use of the intrinsic non-volatility of magnetic tunnel junctions (MTJ) can contribute to mitigate these problems and extend the limits of microelectronics integration. It allows for power gating techniques, which consist in cutting-off the power supply of unused parts of the circuit to reduce leakage. However other functionalities can also be implemented making use of the magnetic tunnel junctions as magnetic field sensors, or using spin-transfer torque (STT) effect to achieve RF generation and detection in the $\mathrm{GHz}$ range, as well as very compact implementation of random number generators.

\section{A. Memory operation \\ MRAM memory and CMOS logic}

In our modified perpendicular anisotropy pMTJ stack, the thermal stability estimated from the switching field distributions, was higher than $40 \mathrm{k}_{\mathrm{B}} \mathrm{T}$, which is a minimum value to obtain 10 year stability, that also depends on the total number of bits. This retention can be adjusted by playing on the cell area, meaning that their stability, and correlatively their switching voltage can be defined by design. This effect appears only because the storage thickness choice allows for the reduction of domain wall nucleation sites for smaller cell area and circumference. Efficiency of STT writing is $1.5 \mathrm{k}_{\mathrm{B}} \mathrm{T} / \mu \mathrm{A}$, for a $30 \mathrm{~nm}$ diameter device, about half the value of state-ofthe art memory dedicated stacks [1].

\section{B. Non-volatile logic circuits}

Non-volatility in logic allows for a local and immediate backup of the data before going into sleep mode, to implement normally-off/instant-on computing. Depending on the application, if the ratio between the activity and inactivity periods is large enough, the gain in leakage reduction compensates the energy required to backup the data in the MTJs yielding a strong overall power reduction. Based on the demonstrated MRAM memory performance, a full hybrid CMOS/Magnetic System on Chip was designed [2] and the corresponding silicon demonstrator was fabricated in $130 \mathrm{~nm}$ technology node (Fig. 4). The gain in power consumption was extrapolated for advanced technology nodes (28nm FDSOI) in [3] and evaluated by simulation. The results show that a gain is obtained as soon as the sleep duration is above $500 \mu \mathrm{s}$.

\section{Magnetic field sensing and RF spin torque oscillators}

Magnetic field sensor operation relies on junction tunnel magnetoresistance (TMR) change as the relative angle between reference and sensing layer magnetization $\theta$ is modified by an external field. The conductance varies as $\cos (\theta)$. Two sensing configurations can be implemented. The first one is for predominantly vertical magnetic fields. For a cell initialized in the anti-parallel state, both the storage layer and top SAF reference layer rotate at different rates under the applied field. The second configuration is more adapted to sense mostly inplane field. In this case, the storage layer will rotate away from the vertical axis. To achieve higher sensitivities, one can introduce magnetic flux concentrators (MFC) made of electrodeposited $\mathrm{NiFe}$ on either side of the magnetic tunnel junction. The field amplification factor can be designed adjusting the NiFe thickness $(2.5-4.5 \mu \mathrm{m})$ and gap $(5-11 \mu \mathrm{m})$. A maximum amplification of $6 \times$ was obtained for the highest thickness/gap ratio. Fig. 7 reports the sensitivity and range of each sensor configuration. A reduction of the offset field through process optimization is still required and desirable for both sensor and memory operation.

Magnetic tunnel junctions also allow for radio frequency (RF) functionality, converting a DC current into RF voltage (signal generation) or converting an RF signal into a DC voltage (signal detection). Both functionalities rely on the spin transfer torque that can drive self-sustained magnetization oscillations under strong enough DC current or excite damped eigenmodes of the magnetization under RF current. The magnetization oscillations are converted via the TMR into a voltage signal. Examples for both functionalities are shown for one pMTJ device in Fig. 8 under an out-of plane field. Signal generation occurs for moderate values of DC current $(<1 \mathrm{~mA})$ with frequencies in the range of $3-5 \mathrm{GHz}$ (Fig. 8a,b), a minimum linewidth of $20 \mathrm{MHz}$ and a maximum power of $20 \mathrm{nW}$. The large tuning of frequency with current allows for digital frequency modulation up to $\mathrm{f}_{\bmod }=20 \mathrm{MHz}$, shown in Fig. 8c for which an additional modulation signal is added at the input of the MTJ device. Fig. 8d shows the RF-to-DC conversion, where a DC output signal appears, when the frequency of the excitation signal is close to the frequency of an eigenmode. DC voltage levels of $10 \mu \mathrm{V}$ to $10 \mathrm{mV}$ were obtained for RF power levels of $-40 \mathrm{dBm}$ to $-5 \mathrm{dBm}$. These RF 
properties will be of interest for wireless communication schemes used within IoT applications.

\section{Acknowledgment}

This work received funding from the European Union's Horizon 2020 research and innovation program under grant GREAT No. 687973 and ERC Advanced Grant MAGICAL No. 669204.

\section{References}

[1] L. Thomas et al., J. Appl. Phys., vol. 115, p. 172615, Apr. 2014

[2] S. Senni et al., 2018 IFIP/IEEE International Conference on VLSISoC, Verona, Italy, 2018, pp. 188-191

[3] G. Patrigeon et al., IEEE Access, vol. 7, pp. 58085-58093, 2019
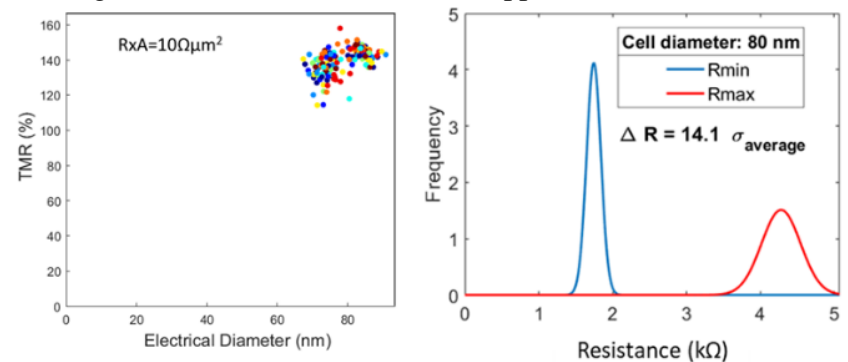

Fig. 1 Multifunctional perpendicular anisotropy stack with $140 \%$ TMR. Plot of low-high resistance states of $80 \mathrm{~nm}$ diameter MRAM cells as obtained in a full dye.
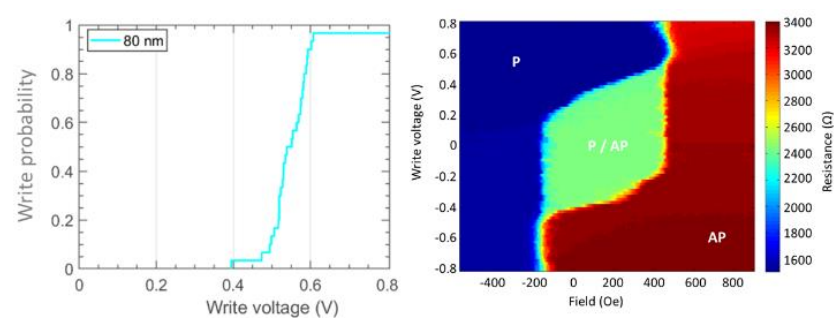

Fig. 2 Memory writing at $0.6 \mathrm{~V}$ for $100 \mathrm{~ns}$ pulses and resistance state diagram as function of pulse voltage and perpendicular applied field.
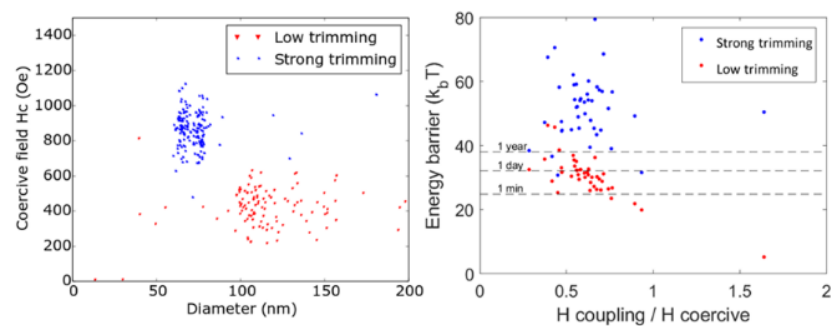

Fig. 3 Cell stability and coercivity defined by cell trimming process.

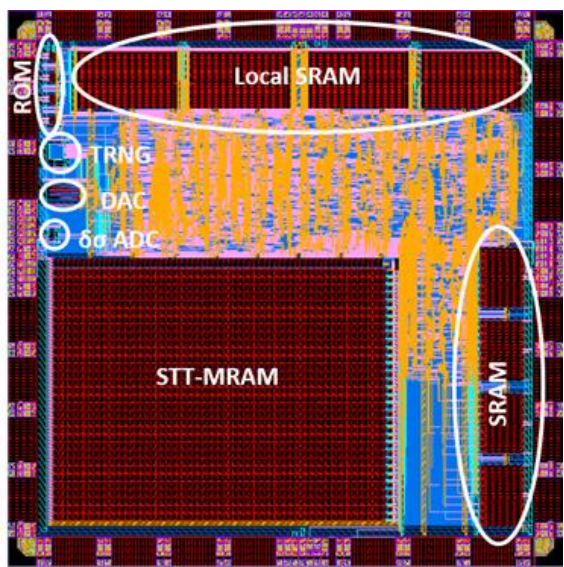

Fig. 4 Non-volatile hybrid CMOS/magnetic SoC demonstrator on
TowerJazz 130nm hybrid process, to validate MRAM based nonvolatile flip-flop/registers. Magnetic devices used for true random number generators, converters and analog sensor functions.

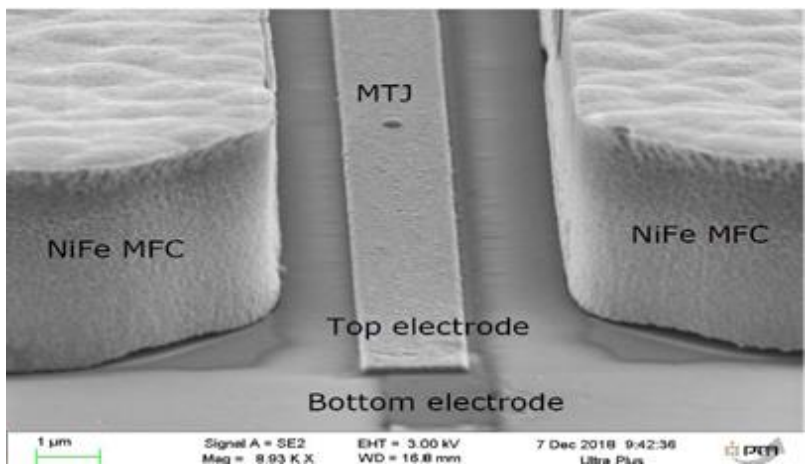

Fig. 5 Tunnel junction sensor including magnetic flux concentrators.
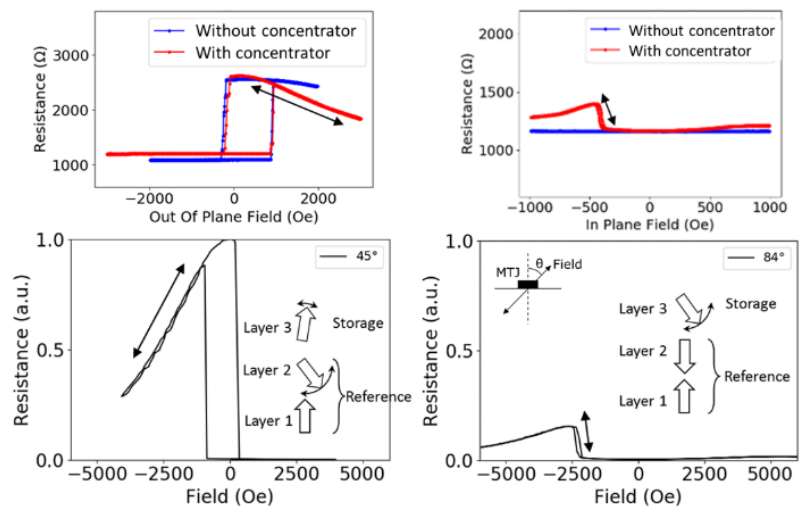

Fig. 6 Magnetoresistance loops of a perpendicular MTJ and operation modes in perpendicular and in-plane field configurations.

\begin{tabular}{|l|c|c|}
\hline & Perpendicular & In-plane \\
\hline Field angle $\theta$ & 10 to $45^{\circ}$ & 80 to $90^{\circ}$ \\
\hline Sensitivity & 0.05 to $0.08 \% / \mathrm{mT}$ & 1 to $10 \% / \mathrm{mT}$ \\
& 0.15 to $0.25 \mathrm{~V} / \mathrm{T}$ & 4 to $40 \mathrm{~V} / \mathrm{T}$ \\
\hline Range & $1500 \mathrm{Oe}$ & $50 \mathrm{Oe}$ \\
\hline
\end{tabular}

Fig. 7 Magnetic sensor characteristics in perpendicular and in-plane field. The sensitivity in $\mathrm{V} / \mathrm{T}$ was estimated with $400 \mathrm{mV}$ bias voltage.
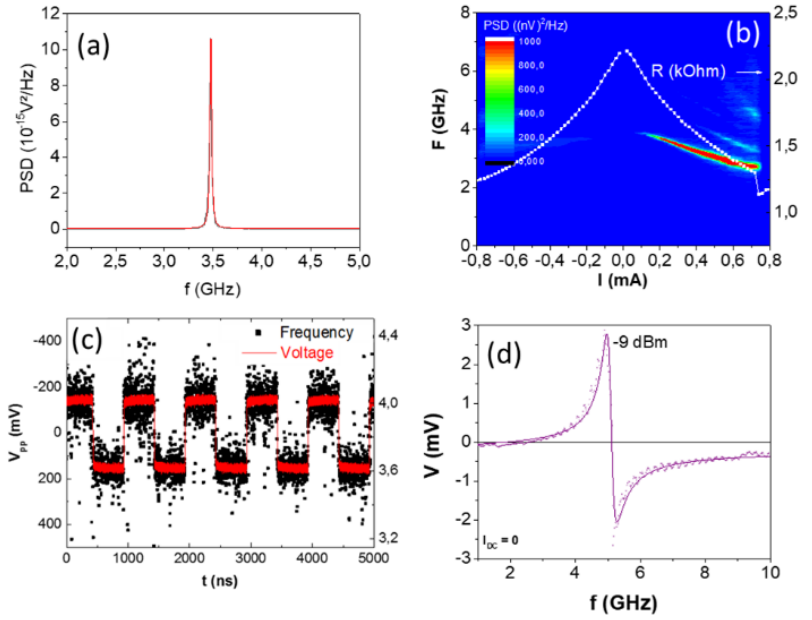

Fig. 8 RF functionality for signal generation a) power spectral density plot vs frequency. b) Frequency tuning vs. DC current. c) digital frequency modulation for $1 \mathrm{MHz}$ modulation frequency and $300 \mathrm{mV}$ modulation voltage creating a $400 \mathrm{MHz}$ shift. d) RF signal detection with $300 \mu \mathrm{V}$ peak-peak DC voltage at $\sim 5 \mathrm{GHz}$ upon injection an RF signal at $-9 \mathrm{dBm}$ power level. 